\section{FP1 Kansas Team Nutrition}

Tessa Adcock, MS, RD, LDN, tadcock@ksde.org, Kansas State Department of Education, Child Nutrition \& Wellness, Landon State Office Building, 900 SW Jackson Street, Suite 251, Topeka, KS, 66612; E. Brinkman, $R D, L D$

Objective: To collaborate with nutrition and wellness stakeholders for maximum outreach to students, school administrators and staff, foodservice professionals, parents, and the community about projects conducted within the Team Nutrition Training Grant received by the Kansas State Department of Education.

Description: Focused on the collaborative aspect of a wellness community, the Kansas Team Nutrition projects implemented within these grants revolve around the expertise, contribution, and expansive outreach of our partners. The modes of nutrition education materials ranged from multi-media creation, newsletters to a targeted population, recipe formulation, curriculum development, state-wide promotions, evaluation tool development, and professional development opportunities.

Evaluation: Resulting final products from each partnership and project goal reached a unique, targeted population. Kansas State Department of Education resources have been extended to over 18,700 students through nutrition education promotion, 4,000 foodservice professionals in training classes developed and 51,150 Kansas households through newsletter outreach with the Team Nutrition Training Grants.

Conclusions and Implications: Building partnerships and collaborating with a variety of organizations positively impacts the quality and reach of nutrition education materials designed with Team Nutrition Training Grant funds.

Funding: USDA Team Nutrition Training Grants \#CNTN14-KS(C), \#CNTN-15-KS(C)

\section{FP2 Scratch Cooking and Smart Snacks: A Minnesota Team Nutrition Recipe Book}

Gail Anderson, $M P H, R D$, gail.c.anderson@state.mn.us, Minnesota Department of Education, 1500 Highway 36 West, Roseville, MN 55113

Objective: To support schools in meeting the USDA Smart Snacks in Schools guidelines through chef developed recipes that use scratch cooking techniques and are compliant with USDA standards.

Description: The Smart Snacks regulation impacts the food that schools sell on their school campus. Scratch cooking is an effective way to manage nutrient content in foods and school food service staff have become increasingly interested in scratch cooking. School food service staff have requested recipes that can be sold under Smart Snacks guidelines that already have the nutrient analysis information determined. Additionally, Food and Consumer Science (FCS) teachers are seeking recipes to engage students in healthy cooking activities and have expressed interest in using recipes that are consistent with the Smart

\section{USDA FNS Poster Abstracts}

Snacks guidelines. A resource available to both school food service and FCS classes will strengthen collaboration across school programs.

Evaluation: Minnesota school food service staff were surveyed on their current practices and opinions related to scratch cooking and recipes for items that can be sold during the schools day. Results are being used to inform a recipe project.

Conclusions and Implications: Recipes meeting Smart Snacks guidelines will be developed by a chef and tested in schools. The recipes will be available to school food service and FCS teachers, assisting them in meeting regulations with student-tested recipes.

Funding: USDA Team Nutrition Training Grant \#CNTN$15-\mathrm{MN}$

\section{FP3 Outcomes From a Montana School Wellness in Action Mini-Grant Program and Implications for Sustaining Long-Term Policy Support}

Katie Bark, RD,LN, SNS, kbark@mt.gov, Montana State University, Montana Team Nutrition Program, 325 Reid Hall, Bozeman, MT 59717; M. Stenberg, RDN, LN

Objective: To provide a financial incentive to support the implementation of the federally mandated school wellness policy in Montana school districts. To learn effective methods and best practices that school districts utilize for building and sustaining a healthy school nutrition environment.

Description: By analyzing data from the final reports and follow-up surveys from three cycles of the School Wellness in Action mini-grant program, a summary of wellness projects, leadership methods and specific outcomes was developed. Key factors, best practices, and implications for long-term implementation of the local wellness policy and/or sustainability of a student wellness initiative were identified.

Evaluation: A 3 year analysis of the final report and follow-up survey of the school wellness mini grant program identified effective methods used to lead and carry out projects, mini-grant outcomes, and the implications for long-term support of the school wellness policy implementation in school districts in a rural state.

Conclusions and Implications: The distribution of funds through a competitive mini-grant program is an effective method for promoting student wellness programs in rural school districts. Supporting a part-time school wellness coordinator is a best practice to yield maximum impact. Use of funds to kick start a wellness initiative can also build long-term support if grassroots support is also strengthened. Farm to school programs are a growing area of interest for nutrition education and promotion in Montana schools. While long term access to funding can be a challenge, collective impact can be produced by 